\title{
Five-week dietary exposure to dry diets alters the faecal bacterial populations in the domestic cat (Felis catus)
}

\author{
Emma N. Bermingham ${ }^{1 *}$, Sandra Kittelmann ${ }^{2}$, Gemma Henderson $^{2}$, Wayne Young ${ }^{1}$, Nicole C. Roy ${ }^{1,3}$ \\ and David G. Thomas ${ }^{4}$ \\ ${ }^{1}$ Food Nutrition Genomics Team, Food and Textiles Group, Agri-Food and Health Section, AgResearch Grasslands, \\ Palmerston North 4442, New Zealand \\ ${ }^{2}$ Rumen Nutrition and Microbiology, AgResearch Grasslands, Palmerston North 4442, New Zealand \\ ${ }^{3}$ The Riddet Institute, Massey University, Palmerston North 4442, New Zealand \\ ${ }^{4}$ Centre of Feline Nutrition, Massey University, Palmerston North 4442, New Zealand \\ (Received 20 October 2010 - Revised 10 November 2010 - Accepted 8 December 2010)
}

\section{Abstract}

The effects of wet (canned) or dry (kibbled) diets on faecal bacterial populations in the cat were investigated in eight domestic short-haired cats (four males and four females; averaging 6 years of age and $3.4 \mathrm{~kg}$ ) in a nested design. The cats were fed ad libitum a commercially available wet diet (moisture $82.0 \%$, crude protein $51.7 \%$, fat $28.9 \%$, carbohydrate (CHO) $8.9 \%$ and ash $10.6 \%$ DM) for 5 weeks. On the fifth week, individual feed intakes and faecal outputs were determined. Fresh faecal samples were collected twice daily, mixed for homogeneity, subsampled and stored at $-85^{\circ} \mathrm{C}$ until analysis. The cats were then switched to a commercially available dry diet (moisture $8.5 \%$, crude protein $33.0 \%$, fat $11.0 \%$, CHO $49.4 \%$ and ash $6.6 \% \mathrm{DM})$ for 5 weeks, and fresh faeces were sampled as described previously. Energy intake tended to be higher in cats fed dry diets $(P<0 \cdot 10)$, but body weight was similar between the two feeding periods $(P>0 \cdot 05)$ Denaturing gradient gel electrophoresis (DGGE) of bacterial 16S rRNA genes amplified from DNA extracted from faeces was performed. The unweighted pair group method with arithmetic mean cluster analysis of bacterial community profiles using Pearson's correlation revealed diet-specific clustering when the same cats were fed on either a dry or a wet diet (dissimilarity between the groups, $88.6 \%$; $P<0 \cdot 001)$. Subsequent cloning and sequencing of five selected distinct DGGE bands indicated that members of the Pelomonas and Fusobacteriaceae were influenced by a short-term change in diet format. This suggests that 5-week dietary exposure is sufficient to alter gastrointestinal microflora.

Key words: Diet format: Domestic cats: Faecal bacterial communities

Reports have suggested that obesity levels in domestic cats are between 25 and $40 \%{ }^{(1)}$ and are increasing ${ }^{(2)}$. Despite being obligate carnivores, domestic cats are consuming highly palatable, carbohydrate $(\mathrm{CHO})$-rich diets $(30-60 \%$ on a DM basis $)^{(3)}$. Domestic cats fed ad libitum on high-CHO, dry diets gained $1.5-2.0 \mathrm{~kg}$ in 16 weeks $^{(4)}$. Dry diets have a low protein: $\mathrm{CHO}$ ratio, which has been linked to obesity in cats $^{(5)}$ and human subjects ${ }^{(6)}$. While cats have a limited ability to digest dietary $\mathrm{CHO}^{(7,8)}$, high dietary $\mathrm{CHO}$ levels, such as those found in dry diets, may result in a level of high-CHO load reaching the intestine. The impacts of this on intestinal microbiota are unknown; however, changes in dietary protein and fibre alter the major species in the feline intestinal tract, including Clostridium perfringens ${ }^{(9)}$, Fusobacterium ${ }^{(9-12)}$, Bifidobacterium $^{(9)}$, Escherichia coli ${ }^{(10)}$ and Lactobacillus populations $^{(10)}$.

We hypothesise that the increase in $\mathrm{CHO}$ load associated with feeding of dry diets may alter the microbial populations within the digestive tract of the domestic cat. We investigate this hypothesis using faecal microbial populations as a representation of gastrointestinal microbiota. The aim of the present study was to investigate the effects of a 5-week dietary exposure to wet and dry diets on the faecal microbial population in domestic cats.

Abbreviations: AAFCO, Association of American Feed Control Officials; CHO, carbohydrate; DGGE, denaturing gradient gel electrophoresis; HDA, heteroduplex diversity assay; UPGMA, unweighted pair group method with arithmetic mean.

*Corresponding author: E. N. Bermingham, fax +646351 8003, email emma.bermingham@agresearch.co.nz 


\section{Materials and methods}

\section{Animals and diets}

The protocol for the present study was approved by the Massey University Animal Ethics Committee (MUEAC no. 09/103). All cats were housed at the Centre for Feline Nutrition (Massey University, Palmerston North, New Zealand) according to the Animal Welfare (Companion Cats) Code of Welfare (2007). Before the study, all cats had complete blood counts, and thyroid assessment was carried out to ensure each cat was clinically and physiologically healthy.

The effects of wet (canned) or dry (kibbled) diets on the faecal bacterial communities of cats were investigated in eight domestic short-haired cats (four males and four females; averaging 6 years of age and $3.4 \mathrm{~kg}$ at the start of the study) using a nested design. The cats were housed in colony cages $(1400 \mathrm{~cm} \times 2400 \mathrm{~cm} \times 4400 \mathrm{~cm})$ and fed ad libitum a commercially available, Association of American Feed Control Officials (AAFCO, Atlanta, GA, USA) tested, wet diet (82.0\% moisture), which provided complete and balanced nutrition (crude protein $51.7 \%$, fat $28.9 \%$, CHO $8.9 \%$ and ash $10.6 \%$ DM) for the maintenance of adult cats, for 5 weeks. On the fifth week, the cats were placed in individual cages $(80 \mathrm{~cm} \times 80 \mathrm{~cm} \times 110 \mathrm{~cm})$ for $5 \mathrm{~d}$ to determine individual feed intakes and faecal outputs. Fresh faecal samples were collected twice daily, mixed for homogeneity, subsampled and stored at $-85^{\circ} \mathrm{C}$ until analysis. The process was then repeated for a commercially available, AAFCO-tested, dry diet ( $8.5 \%$ moisture), which also provided complete and balanced nutrition (crude protein $33.0 \%$, fat $11.0 \%$, $\mathrm{CHO}$ $49.4 \%$ and ash $6.6 \%$ DM) for the maintenance of adult cats. Energy content of the diet was determined using the metabolisable energy protocol outlined by the AAFCO ${ }^{(13)}$

(a)

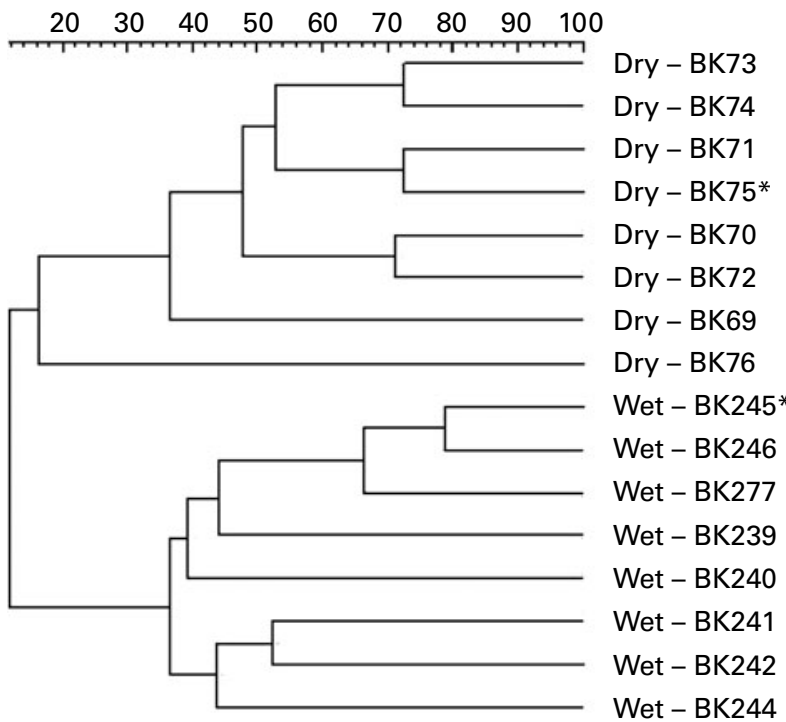

\section{Microbial community analysis of cat faeces} \\ Nucleic acids were extracted from $30 \mathrm{mg}$ of faeces with a \\ combined bead-beating and phenol-chloroform protocol ${ }^{(14)}$. \\ After bead beating with a FastPrep FP120 (Qbiogene, Carlsbad, \\ CA, USA), cells were chemically disrupted with phenol- \\ chloroform-isoamyl alcohol (25:24:1) and chloroform-isoa- \\ myl alcohol (24:1). DNA was precipitated from the aqueous \\ phase with polyethylene glycol (30\%). The DNA pellet was \\ washed with $70 \%$ ice-cold ethanol, dried and resuspended in \\ $100 \mu \mathrm{l}$ of molecular biology grade water. Extracted DNA was \\ quantified with a NanoDrop ND-1000 spectrophotometer \\ (NanoDrop Technologies, Wilmington, DE, USA), quality \\ checked on a $1 \%$ agarose gel and the $\mathrm{V} 2-\mathrm{V} 3$ regions of bacterial \\ $16 \mathrm{~S}$ rRNA genes were amplified from each sample using primers \\ heteroduplex diversity assay (HDA)1-GC and HDA-2 ${ }^{(15)}$. PCR \\ products were quality checked on $2 \%(\mathrm{w} / \mathrm{v})$ agarose gels to \\ confirm a single band of approximately $200 \mathrm{bp}^{(16)}$. Products \\ were purified using a MinElute PCR purification kit (Qiagen, \\ Valencia, CA, USA) followed by digestion with Mung Bean \\ nuclease (Promega, Madison, WI, USA) to remove single- \\ stranded DNA. Denaturing gradient gel electrophoresis \\ (DGGE) was performed using the Temporal Temperature \\ Gel Electrophoresis System (CBS Scientific Company, Inc., \\ Del Mar, CA, USA). The $6 \%$ polyacrylamide gel contained a \\ denaturing gradient of $35-70 \%$ (100\% denaturant was $7 \mathrm{M}$-urea \\ and $40 \%(\mathrm{v} / \mathrm{v})$ formamide $)^{(16)}$. Electrophoresis was performed \\ at constant voltage $(65 \mathrm{~V})$ and temperature $\left(60^{\circ} \mathrm{C}\right)$ for $14 \mathrm{~h}$. \\ The gel was stained with SYBR Gold (Invitrogen, Carlsbad, \\ CA, USA) and visualised by UV transillumination (Fig. 1(a)). \\ We selected five distinct bands based on their presence or \\ absence from each diet and excised from a representative \\ sample of each treatment group (Fig. 1(b)) in order to phylo-
}

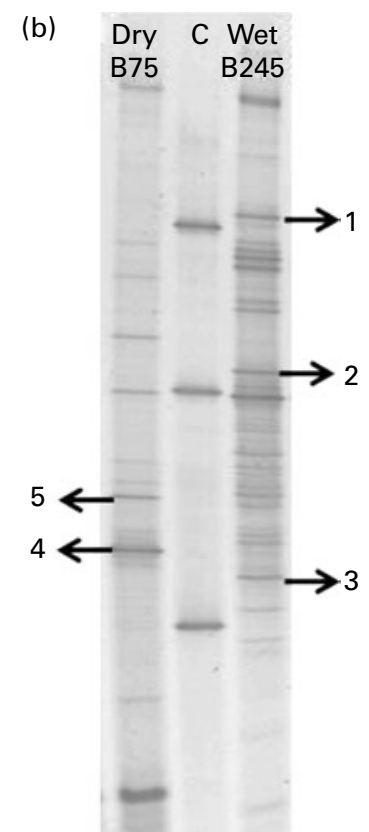

Fig. 1. (a). Dendrogram showing similarities of bacterial 16S rRNA gene community profiles obtained by denaturing gradient gel electrophoresis (DGGE) fingerprinting from the faeces of cats fed wet or dry diets. Samples denoted by an asterisk (*) were used for the excision of bands. (b) Exemplary DGGE profiles from cats fed wet or dry diets. Arrows indicate the positions, where bands were excised (based on their presence or absence from each diet), and DNA was recovered and sequenced. Numbers match the phylogeny assignments indicated in Table 1. A control marker (C) was applied as an external standard. 
genetically assign some of the bacterial populations potentially affected by the diet shift. DNA eluted from the band underwent PCR using HDA1/2 ${ }^{(16)}$. The resultant PCR products were purified (as described earlier) and cloned using the TOPO-TA cloning kit (Invitrogen). The inserts were amplified from the plasmids using HDA1-GC/HDA-2 primers $^{(16)}$, and correct band migration was verified by DGGE. DNA sequencing of clones was performed at the Allan Wilson Centre Genome Service (Massey University, Palmerston North, New Zealand) using a capillary ABI3730 Genetic Analyser (Applied Biosystems, Inc., Foster City, CA, USA), and sequence information was evaluated using the basic local alignment search tool (BLAST) ${ }^{(17)}$.

\section{Bionumerics and statistics}

Digital images of DGGE were analysed using the bandsearching algorithm of the Bionumerics software (Applied Maths, Austin, TX, USA). The unweighted pair group method with arithmetic mean (UPGMA) cluster analysis of bacterial community profiles was performed using Pearson's correlation. A position tolerance of band matching of $1 \%$ was used for all analyses. Energy intake and body weight were assessed for normality and the presence of outliers by plotting residuals $v$, the predicted residuals, and analysed using a general linear model (Minitab version 15; Minitab Limited, Coventry, UK), using diet type as the main factor of interest. Results are reported as means with their standard errors of the mean, and were considered significant at $P<0.05$ and a trend at $P>0.05$ and $P<0 \cdot 10$.

\section{Results}

Energy intake tended $(P<0 \cdot 10)$ to be higher in cats fed the dry diets (dry $467 \cdot 7 v$. wet $397 \cdot 0(\operatorname{sem} 25 \cdot 10) \mathrm{kJ} / \mathrm{kg}$ body weight per d). Body weight was similar $(P>0.05)$ between the two feeding periods (dry $3.6 v$. wet 3.4 (SEm 1.4 ) kg).

Changes in the bacterial communities in the faeces of cats fed wet and dry diets were assessed with DGGE. UPGMA cluster analysis of bacterial community profiles using Pearson's correlation revealed diet-specific clustering when the same cats were fed on either a dry or a wet diet (dissimilarity between the groups, 88.6\%; $P<0 \cdot 001$; Fig. 1(a)). Sequencing of selected bands (Fig. 1(b)) showed that, among others, the presence of individual species from the Fusobacteriaceae and Pelomonas species was changed by the dietary format (Table 1).

\section{Discussion}

We identified a significant shift in faecal bacterial communities induced by a 5-week dietary exposure to dry and wet diets in the domestic cat. Changes in bacterial populations included alterations in the Fusobacteriaceae and Pelomonas species, which are known to be present within the feline digestive tract ${ }^{(18,19)}$; however, their metabolic role is not clear. The present results are in agreement with recent studies using quantitative PCR which have shown that changes in the macronutrient content of the diet alter faecal microbial populations in the domestic cat. For example, high-protein diets increase the levels of $C$. perfringens and Fusobacterium species and decrease Bifidobacterium populations in the faeces of adult cats ${ }^{(9)}$. In weaned kittens, high dietary protein decreased E. coli, Bifidobacterium and Lactobacillus populations ${ }^{(10)}$. Changes in dietary fibre also affect the major bacterial populations in the domestic cat, including the Fusobacterium species ${ }^{(11,12)}$.

The cats in the present study were fed either a commercially available wet or a dry diet. While cats have evolved as strict carnivores with little or no $\mathrm{CHO}$ in their diets, the dry diet in the present study contained high (50\% DM) levels of CHO compared with the wet diet $(9 \%$ DM). This dry diet had a low protein: $\mathrm{CHO}$ ratio, which has been linked to obesity in cats ${ }^{(5)}$ and humans ${ }^{(6)}$. The use of $\mathrm{CHO}$ in feline nutrition is a contentious issue, with debate about the long-term impacts on feline health due to high dietary $\mathrm{CHO}$ levels ${ }^{(20)}$. The shifts in the faecal microbiota observed in the present study may be as a result of an increased CHO load entering the large intestine due to the low protein: $\mathrm{CHO}$ ratio in the dry diets.

Emerging evidence suggests that obesity is more than a disturbance of tissue metabolism and energy intake, and that microbiota are critical to the development of obesity ${ }^{(21,22)}$

Table 1. List of clones that were obtained from excised DGGE bands along with their closest BLAST hits (excluding environmental clone sequences)

Closest cultivated relative using BLAST

\begin{tabular}{lccllc}
\cline { 3 - 5 } Band $^{*}$ & Clone & Diet & Accession no. & Species & $\begin{array}{c}\text { Maximum } \\
\text { identity (\%) }\end{array}$ \\
\hline 1 & 1 & Wet & GU797848 & Fusobacterium sp. 3019/04 & 99 \\
& 2 & & EU728722 & Fusobacteriaceae bacterium DJFB254 & 95 \\
2 & 1 & Wet & AM989106 & Pelomonas sp. AKB-2008-ET9 & 99 \\
& 2 & & AM989106 & Pelomonas sp. AKB-2008-ET9 & 99 \\
3 & 1 & Wet & EU728722 & Fusobacteriaceae bacterium DJFB254 & 95 \\
& 2 & & EU728722 & Fusobacteriaceae bacterium DJFB254 & 95 \\
4 & 1 & Dry & GU797848 & Fusobacterium sp. 3019/04 & 99 \\
& 2 & & GU797848 & Fusobacterium sp. 3019/04 & 99 \\
5 & 1 & Dry & AM989106 & Pelomonas sp. AKB-2008-ET9 & 99 \\
& 2 & & AM989106 & Pelomonas sp. AKB-2008-ET9 & 100 \\
& 3 & & AM989106 & Pelomonas sp. AKB-2008-ET9 & 100 \\
\hline
\end{tabular}

* Number of bands refer to Fig. 1. 
Therefore, the development of obesity may be in part due to alterations in the microbial populations within the digestive tract of the domestic cat.

The present study shows that the changes that occurred in the faecal microbial population in domestic cats as a result of diet format were numerous but often subtle and therefore difficult to describe on a taxonomic level by means of DGGE alone. Alternative methods such as next-generation sequencing may provide a much more detailed insight into the effects of diet changes on the bacterial communities in domestic cats.

\section{Acknowledgements}

We acknowledge the assistance of Dr Shalome Basset (AgResearch), Ms Karin Weidgraaf and Margreet Hekman (Massey University) in the present study. The present study was funded by the Massey University Research Fund (09/0240). There are no conflicts of interest. E. N. B., N. C. R. and D. G. T. obtained funding, and designed and conducted the animal study. E. N. B., W. Y., S. K. and G. H. designed and conducted the laboratory component of the study. All authors contributed to the writing of the manuscript.

\section{References}

1. Scarlett JM, Donoghue S, Saidla J, et al. (1994) Overweight cats: prevalence and risk factors. Int J Obes Relat Metab Disord 18, S22-S28.

2. German AJ (2006) The growing problem of obesity in dogs and cats. J Nutr 136, S1940-S1946.

3. de-Oliveira LD, Carciofi AC, Oliveira MC, et al. (2008) Effects of six carbohydrate sources on diet digestibility and postprandial glucose and insulin responses in cats. J Anim Sci 86, 2237-2246.

4. Weidgraaf K, Tucker LA \& Thomas DG (2007) A link between diet form and weight change in domestic cats (Felis catus)? Asia Pac J Clin Nutr 16, S110.

5. Vester BM, Liu KJ, Keel TL, et al. (2009) In utero and postnatal exposure to a high-protein or high-carbohydrate diet leads to differences in adipose tissue mRNA expression and blood metabolites in kittens. Br J Nutr 102, 1136-1144.

6. Simpson SJ \& Raubenheimer D (2005) Obesity: the protein leverage hypothesis. Obes Rev 6, 133-142.

7. Kienzle E (1993) Carbohydrate metabolism of the cat. 1. Activity of amylase in the gastrointestinal tract of the cat. J Anim Physiol Anim Metab 69, 91-101.
8. Kienzle E (1994) Effect of carbohydrates on digestion in the cat. J Nutr 124, 2568S-2571S.

9. Lubbs DC, Vester BM, Fastinger ND, et al. (2009) Dietary protein concentration affects intestinal microbiota of adult cats: a study using DGGE and qPCR to evaluate differences in microbial populations in the feline gastrointestinal tract. J Anim Physiol Anim Nutr 93, 113-121.

10. Vester BM, Dalsing BL, Middelbos IS, et al. (2009) Faecal microbial populations of growing kittens fed high- or moderate-protein diets. Arch Anim Nutr 63, 254-265.

11. Barry KA, Wojcicki BJ, Middelbos IS, et al. (2010) Dietary cellulose, fructooligosaccharides, and pectin modify fecal protein catabolites and microbial populations in adult cats. J Anim Sci 88, 2978-2987.

12. Bueno AR, Cappel TG, Sunvold GD, et al. (2000) Feline colonic microbes and fatty acid transport: effects of feeding cellulose, beet pulp and pectin/gum arabic fibers. Nutr Res 20, 1319-1328.

13. AAFCO (2009) Official Publication. Atlanta, GA: Association of American Feed Control Officials.

14. Kittelmann S \& Janssen PH (2010) Characterisation of rumen ciliate community composition in domestic sheep, deer, and cattle feeding on varying diets, by means of PCR-DGGE and clone libraries. FEMS Microbiol Ecol 75, 468-481.

15. Tannock GW (2000) The intestinal microflora: potentially fertile ground for microbial physiologists. Adv Microb Physiol 42, 25-46.

16. Knoch B, Nones K, Barnett MPG, et al. (2010) Diversity of cecal bacteria is altered in interleukin-10 gene-deficient mice before and after colitis onset and when fed polyunsaturated fatty acids. Microbiology 156, Pt 11, 3306-3316.

17. Altschul SF, Gish W, Miller W, et al. (1990) Basic local alignment search tool. J Mol Biol 215, 403-410.

18. Ritchie LE, Steiner JM \& Suchodolski JS (2008) Assessment of microbial diversity along the feline intestinal tract using $16 \mathrm{~S}$ rRNA gene analysis. FEMS Microbiol Ecol 66, 590-598.

19. Ritchie LE, Burke KF, Garcia-Mazcorro JF, et al. (2010) Characterization of fecal microbiota in cats using universal $16 \mathrm{~S}$ rRNA gene and group-specific primers for Lactobacillus and Bifidobacterium spp. Vet Microbiol 144, 104-114.

20. Laflamme DP (2008) Letter to the Editor: cats and carbohydrates. Top Companion Anim Med 23, 159-160.

21. Turnbaugh PJ, Hamady M, Yatsunenko T, et al. (2009) A core gut microbiome in obese and lean twins. Nature $\mathbf{4 5 7}$, 480-484.

22. Turnbaugh PJ, Ley RE, Mahowald MA, et al. (2006) An obesity-associated gut microbiome with increased capacity for energy harvest. Nature 444, 1027-1031. 